\title{
Relationship Between Metabolic Syndrome and Serum Uric Acid in an Iranian Community Elderly Population: A Cohort Aging Study
}

\author{
Mansour Babaei ${ }^{1,2}$, Afsaneh Bakhtiari ${ }^{*}$, Elham Divband ${ }^{3}$, Ali Bijani ${ }^{4}$, Seyed Reza Hosseini ${ }^{4}$ \\ ${ }^{1}$ Mobility Impairment Research Center, Health Research Institute, Babol University of Medical Sciences, Babol, Iran \\ ${ }^{2}$ Department of Internal Medicine, School of Medicine, Babol University of Medical Sciences, Babol, Iran \\ ${ }^{3}$ Clinical Research Development Center, Ayatollah Rohani Hospital, Babol University of Medical Sciences, Babol, Iran \\ ${ }^{4}$ Social Determinants of Health Research Center, Health Research Institute, Babol University of Medical Sciences, Babol, Iran
}

Corresponding Author: Afsaneh Bakhtiari, Mobility Impairment Research Center, Health Research Institute, Babol University of Medical Sciences, Babol, Iran. Tel: +98-9113116889, Email: afbakhtiari@gmail.com

Received March 15, 2019; Accepted June 26, 2019; Online Published September 12, 2019

\begin{abstract}
Introduction: Serum uric acid (UA) level is reported to be associated with a variety of cardiometabolic risk factors. Although various studies have reported elevated levels of UA in patients with metabolic syndrome (MetS), its clinical interpretation is still controversial and challenging. Thus, we examined the association between hyperuricemia (HUA) and UA levels with MetS, its components and number of components.

Materials and Methods: This population-based cross-sectional study included 1561 older people who participated in Amirkola Health and Aging Project (AHAP) in North of Iran. MetS was defined based on the Iranian National Committee of Obesity criteria. Blood pressure, fasting blood glucose (FBG), lipid profiles, UA and anthropometric measures were determined.

Results: The diagnosis of the MetS was not associated with UA levels and HUA. The results of this study showed that the only FBG is a component which increases the risk of HUA.

Conclusions: Our findings from a community elderly population suggest that the UA levels and HUA were not associated with the diagnosis of the MetS by the stepwise logistic regression approach. However, in spite of various confounding factors, including metabolic and non-metabolic components in the association with MetS and UA, the whole relationship remains to be determined. A large prospective study is needed to reveal the clinical significance of UA in MetS.

Keywords: Metabolic Syndrome, Serum Uric Acid, Aged

Citation: Babaei M, Bakhtiari A, Divband E, Bijani A, Hosseini SR. Relationship between metabolic syndrome and serum uric acid in an Iranian community elderly population: a cohort aging study. J Appl Biotechnol Rep. 2019;6(3):118-124. doi:10.29252/ JABR.06.03.07.
\end{abstract}

\section{Introduction}

Aging is defined as a degenerative process in the biological structure of the body. The process of aging is accompanied by obesity, disorders in fats and sugar metabolism, resistance to insulin, inflammation, and hardening of the artery wall. ${ }^{1,2}$ The prevalence of chronic and non-communicable diseases such as cardiovascular diseases (CVD), hypertension, and diabetes increases with aging. The risk of CVD has been 3 times in the elderly people over the last few years so that $43 \%$ of deaths were due to the CVD in people over 65 years. $^{3}$

Metabolic syndrome (MetS) with the collection of risk factors for CVD was defined as a chronic and progressive disease. ${ }^{4}$ Prevalence of MetS was mentioned 39.7\% in Iran. ${ }^{5}$ The high prevalence of MetS, heart attack and diabetes in the elderly population are caused by age as a risk factor for the metabolic diseases. ${ }^{3}$

Uric acid (UA) is the final product of purine metabolism has been considered as another risk factor for CVD, hypertension, kidney disease and MetS in some studies. ${ }^{6,7}$ $\mathrm{UA}$ is one of the first line antioxidant defense mechanism in the plasma with the roles of a powerful scavenger of single oxygen peroxyl (ROS) and hydroxyl $(\mathrm{OH})$ radicals. ${ }^{7}$ In spite of the antioxidant activity in the extracellular environment, UA has harmful results once it enters vascular smooth muscle cells and adipocytes. ${ }^{8}$ Its effects are inhibition of endothelial function, induction of platelet aggregation, and chronic systemic inflammation. ${ }^{9}$

The relationship between UA level and hyperuricemia (HUA) with clinical conditions such as hypertension, MetS, coronary artery disease, cerebrovascular disease, vascular dementia, gestational hypertension and kidney diseases has been proposed in several epidemiological studies. ${ }^{10-14}$ UA even within the normal range can also be accompanied by CVD, while some authentic centers such as the Institute of

Copyright (C) 2019 The Author(s). This is an open-access article distributed under the terms of the Creative Commons Attribution License (http:// creativecommons.org/licenses/by/4.0), which permits unrestricted use, distribution, and reproduction in any medium, provided the original work is properly cited. 
Framingham believe that UA is not as a risk factor for CVD. ${ }^{15}$

However, the relationship between serum UA levels and the MetS remains controversial. ${ }^{16,17}$ In Iran, several studies have been conducted to examine this relationship, ${ }^{18-21}$ but they have often different methodology through subjects or study designs. Some studies took a wide range of people for age with different health status, ${ }^{18}$ some only in adolescents, ${ }^{19}$ some in patients with coronary artery disease or diabetes, ${ }^{20,21}$ and some just in the middle-aged healthy population. ${ }^{22}$ Although, some studies have shown that UA or HUA is associated with MetS or some of its components, a large population-based study in elderly people may have different results. The reason is some restrictions for the use of UA as an indicator. First, the antioxidative characteristic of UA showed paradoxical value depending on blood concentration. ${ }^{8}$ Second, UA concentration differed with sex..$^{23}$ Third, age may affect UA levels due to aging-related changes in purine metabolism. ${ }^{23}$

Since the combination of HUA and MetS together in the elderly can endanger their health, controlling the level of UA can help improve the health of the elderly. Accordingly, we planned to present the status of UA and prevalence of HUA and their relationship to MetS in the Iranian elderly population. The study results can be helpful in the elderly care intervention programs.

\section{Materials and Methods}

Study Population

This population-based cross-sectional study was derived from the Amirkola Health and Aging Project (AHAP). It is an ongoing cohort study that has been started since 2011 in Amirkola city in the northern part of Iran. The total population of 60 years and over in Amirkola was estimated to be 2234 . A total of 1616 people (73\%) participated in the study. After excluding 56 participants due to missing data on UA level or MetS, 1560 remained in the study. Details of the procedure were published in a previous article. ${ }^{24}$ The project was funded by the Vice-Chancellery of Research and Technology, Babol University of Medical Sciences (BUMS) for investigation of the geriatric medical problems such as falling, bone fragility and fractures, cognitive impairment and dementia, poor mobility and functional dependence. The baseline stage of this project was done in 2011 and 2012. The details of the recruitment of participants were described previously. ${ }^{24}$ The AHAP study was approved by the Medical Ethics Committee of BUMS (MUBABOL.REC.1392.14) and informed consent was obtained from all the participants.

\section{Measurements of Metabolic Syndrome}

The MetS was defined according to the Iranian National Committee of Obesity criteria. ${ }^{25}$ MetS was diagnosed when a person had three or more of the following components: waist circumference (WC) $>95 \mathrm{~cm}$, systolic blood pressure $\geq 130 \mathrm{~mm} \mathrm{Hg}$ or diastolic blood pressure $\geq 85 \mathrm{~mm} \mathrm{Hg}$, highdensity lipoprotein cholesterol (HDL -C) $<40$ in men and $<$ 50 in women, triglyceride $(\mathrm{TG}) \geq 150$, fasting blood glucose $(\mathrm{FBG}) \geq 100 \mathrm{mg} / \mathrm{dL}$, or being treated for low High-density lipoprotein cholesterol (HDL-C) or high blood pressure, TG, and FBG.
In the study, the diagnosis of hypertension was based on blood pressure measurement using the Omron manometer M3 intelligence model in lying position at 2 times using standard methods. Average values of these 2 measurements were considered as hypertension. WC was measured as the width between the bottom rib and the top of the hip of the participants using a metric measuring tape. Weight was measured using a Seca digital scale with minimum clothes, with an accuracy of $0.1 \mathrm{~kg}$, and height was measured by stadiometer with an error of $0.5 \mathrm{~cm}$. Body mass index (BMI) was calculated based on the formula, weight $(\mathrm{kg}) /$ [height $(\mathrm{m})]^{2}$. To measure blood glucose and lipids, after a 12-hour overnight fast, venous blood was drawn and collected in the tubes containing ethylenediaminetetraacetic acid to obtain centrifuged plasma. FBG and lipid profile were measured enzymatically by auto-analyzers. The sampling was done by skilled nurses and analyzed by clinical laboratory experts of Shahid Beheshti hospital of Babol, affiliated to BUMS, Iran.

\section{Measurements of Uric Acid}

The UA was determined on a standard autoanalyzer with Uricase and reagent Parsazmun Co, Tehran, Iran). Levels of UA were created as follows: $4 / 5 \leq \mathrm{mg} / \mathrm{dL}, 4 / 51-6 \mathrm{mg} / \mathrm{dL}, 6 / 01$ $7 \mathrm{mg} / \mathrm{dL}$, and $>7 \mathrm{mg} / \mathrm{dL}$. HUA is defined as an abnormally high level of UA in the serum. In adults, the normal rate is considered more than $6 \mathrm{mg} / \mathrm{dL}$ in women and $7 \mathrm{mg} / \mathrm{dL}$ in men. ${ }^{26}$

\section{Statistical Analysis}

In the present analysis, the key risk factor was MetS and its components, and the outcome was UA levels and HUA. Normality of variables of interest was tested by KolmogorovSmirnov. $\chi^{2}$ and Fisher exact test were used for qualitative variables and ANOVA test for quantitative comparisons.

The relationship between UA and MetS was investigated in three aspects. First, the association between HUA and MetS was investigated in the whole population and based on sex. Then, the relationship between UA levels and MetS components was separately analyzed. Finally, the correlation between HUA and UA levels and MetS was investigated using crude and sex/age-adjusted multiple regression logistic models.

Statistical analysis was performed using SPSS version 18 software (SPSS Inc., Chicago, IL). The two-tailed $P$ value less than 0.05 were considered significant.

\section{Results}

Descriptive Analysis

1560 people participated in the study. A total of 699 (44.7\%) participants were female. The mean age was $68.5 \pm 6.90$ years for women and $69.9 \pm 7.61$ years for men. Table 1 shows the clinical and biochemical characteristics of the elderly population by gender. The prevalence of MetS was $74.36 \%$ $(\mathrm{n}=1160)$ with higher prevalence in women than in men $(85.7 \%$ vs. $65.1 \% ; P<0.001)$. As well as the prevalence of HUA was $5.5 \%$ in the population including $3.13 \%$ in men and $8.44 \%$ in women and it was significantly higher in women than in men $(P<0.001)$. 
Table 1. Clinical and Biochemical Characteristics of the Elderly Population by Gender

\begin{tabular}{|c|c|c|c|}
\hline Variables & Men & Women & $P$ Value \\
\hline MetS & $561(65.1 \%)$ & $599(85.7 \%)$ & 0.001 \\
\hline Hyperuricemia & $27(3.13 \%)$ & $59(8.44 \%)$ & 0.001 \\
\hline $\mathrm{SBP}(\mathrm{mm} \mathrm{Hg})$ & $142.53 \pm 22.1$ & $142.9 \pm 22.2$ & 0.68 \\
\hline DBP (mm Hg) & $81.04 \pm 12$ & $82 \pm 11.6$ & 0.11 \\
\hline $\mathrm{WC}(\mathrm{cm})$ & $95.11 \pm 10.3$ & $96.53 \pm 10.7$ & 0.008 \\
\hline $\mathrm{FBG}(\mathrm{mg} / \mathrm{dL})$ & $114.15 \pm 41.9$ & $122.81 \pm 49.5$ & 0.001 \\
\hline $\mathrm{TG}(\mathrm{mg} / \mathrm{dL})$ & $148.72 \pm 77.6$ & $174.4 \pm 89.4$ & 0.001 \\
\hline HDL-C (mg/dL) & $38.37 \pm 4$ & $39.1 \pm 4.7$ & 0.001 \\
\hline LDL-C (mg/dL) & $122.66 \pm 39.9$ & $137.38 \pm 4.8$ & 0.001 \\
\hline Cholesterol (mg/dL) & $188.34 \pm 39.1$ & $205.59 \pm 44.2$ & 0.001 \\
\hline $\mathrm{BMI}\left(\mathrm{kg} / \mathrm{m}^{2}\right)$ & $26.12 \pm 4$ & $28.49 \pm 4.7$ & 0.001 \\
\hline UA (mg/dL) & $4.93 \pm 0.6$ & $4.70 \pm 0.9$ & 0.001 \\
\hline UA levels & & & 0.001 \\
\hline$\leq 4.5$ & $294(48.84)$ & $308(51.16)$ & \\
\hline $4.51-6$ & $474(58.81)$ & $332(41.19)$ & \\
\hline $6.01-7$ & $67(60.91)$ & $43(39.09$ & \\
\hline$>7$ & $27(62.79)$ & $16(37.21)$ & \\
\hline
\end{tabular}

MetS, metabolic syndrome; SBP, systolic blood pressure; DBP: diastolic blood pressure; FBG, fasting blood glucose; TG, triglycerides; BMI, body mass index, HDL: high-density lipoprotein, WC: waist circumference, LDL: low-density lipoprotein; UA, serum uric acid.

Values shown are means \pm SD or numbers of the participants (percentages).

Table 2. The Association of MetS With HUA by Gender in the Elderly Population

\begin{tabular}{cccc}
\hline \multirow{2}{*}{ MetS } & \multicolumn{2}{c}{ HUA } & P Value \\
\cline { 2 - 3 } & & + & \\
Male & $538(95.9)$ & $23(4.1)$ & 0.03 \\
Yes & $297(98.7)$ & $4(1.3)$ & \\
No & & & 0.03 \\
Female & $554(92.5)$ & $45(7.5)$ & \\
Yes & $86(86)$ & $14(14)$ & 0.29 \\
No & & & \\
Total & $1092(94.1)$ & $68(5.9)$ & \\
Yes & $383(95.5)$ & $18(4.5)$ & \\
No & & \\
\hline
\end{tabular}

MetS, metabolic syndrome; HUA, hyperuricemia.

Values shown are numbers of the participants (percentages).

\section{Acid Uric and Metabolic Syndrome Relationship}

The association between HUA and the prevalence of MetS in the elderly general population had no significant difference, but it showed a significant relationship in both genders $(P=0.026$, in men, and in women $(P=0.031$; Table 2$)$.

The relationship of MetS components with UA levels are shown in Table 3. FBG showed a significant relationship with levels of UA $(P=0.03)$. This means that with increasing levels of UA, the level of FBG also increased, but there was no significant relationship between other MetS components and UA levels.

Correlation between MetS and UA levels within a normal to high range and HUA in logistic models did not show any significant correlation in both crude and adjusted models for age and sex (Table 4).

Figure 1 shows the association of means UA with MetS components number by gender in the elderly population. Mean UA level had no significant relationship with the increase in the number of MetS components in both genders.

\section{Discussion}

The correlation of UA levels and MetS components has yielded conflicting results; while some studies reported strong associations among these variables ${ }^{11,27,28}$ others did not confirm any independent correlation based on multiple regression models. They suggested that this correlation might be gender-specific, limited to higher levels of UA, and limited to a number of MetS components. ${ }^{29-32}$ Similarly, some studies have concluded that the association is confounded by the relationship between UA and established CVD risk factors, including hypertension, obesity, hyperlipidemia, or diabetes mellitus. ${ }^{33-35}$ These findings are consistent with our data which confirmed no correlation between UA/HUA levels and MetS diagnosis using a stepwise logistic regression model in the elderly population. In the present study, the level of UA showed only a significant relationship with FBG, while there was no association with other MetS components and also with the diagnosis of MetS. In support of this finding, Leyva et al study indicated that sensitivity and specificity of HUA for the diagnosis of the MetS were both below $60 \%$ in their study. They concluded that UA levels had no significant predictive power to detect significant MetS.

The role of UA in the diagnosis of MetS and related components was investigated in some earlier studies. In a cohort study conducted among the white male population, UA was found to be loaded on a factor also associated with plasma insulin, FBG, TG and BMI, but not with BP. ${ }^{36}$ In the Community Health Study conducted in Miami among 26 nondiabetic men and 24 women, Donahue et $\mathrm{al}^{37}$ identified a high UA loading on a factor associated with WC, dyslipidemia, insulin resistance, and BP. Hodge et $\mathrm{al}^{38}$ in a study of nondiabetic Mauritians, indicated that UA was related to a BP factor among men, and not in women. Liou et $\mathrm{al}^{39}$ study concluded that UA is aggregated with BMI, WC, FBG, Log TG, and HDL-C and loaded on the metabolic factor. According to our results, UA levels are associated with hyperglycemia. This finding is consistent with those obtained by Viazzi et al,,$^{40}$ Liou et $\mathrm{al}^{39}$ and Aguilar-Andrade et $\mathrm{al}^{41}{ }^{41}$, where the UA levels were identified as a powerful predictor of type 2 diabetes mellitus in primary hypertension, especially in women.

MetS includes a combination of different components and that the different combination of MetS risk factors has different effects on individuals' health. Meigs ${ }^{42}$ reviewed a dozen studies investigating the underlying structure of MetS using factor analysis. They found that MetS was composed of 2-4 domains of phenotypes with the obesity-dyslipidemiahyperinsulinemia domain being its central feature. A separate factor for BP was loosely associated with the core feature. Also, Liou et $\mathrm{al}^{39}$ identified 2 phenotype domains of MetS by factor analysis in the study; a metabolic factor explained onethird of variance, and a BP factor accounted for another $25 \%$ 
Table 3. The Association of MetS Components With UA Levels in the Elderly Population

\begin{tabular}{|c|c|c|c|c|c|}
\hline & \multicolumn{4}{|c|}{ UA levels (mg/dL) } & \multirow{2}{*}{$P$ Value } \\
\hline & $\leq 4.5$ & $4.51-6$ & $6.01-7$ & $>7$ & \\
\hline SBP (mm Hg) & $143.37 \pm 22.4$ & $142.4 \pm 22.2$ & $140.04 \pm 22.2$ & $146.05 \pm 22.6$ & 0.41 \\
\hline FBG (mg/dL) & $112.2 \pm 50.3$ & $115.1 \pm 41$ & $116.2 \pm 52.7$ & $118.1 \pm 35.1$ & 0.03 \\
\hline $\mathrm{HDL}(\mathrm{mg} / \mathrm{dL}$ & $38.55 \pm 4.1$ & $38.78 \pm 4.5$ & $38.6 \pm 3.1$ & $39.3 \pm 5.2$ & 0.58 \\
\hline $\mathrm{TG}(\mathrm{mg} / \mathrm{dL})$ & $161.46 \pm 88$ & $157.91 \pm 79.9$ & $161 \pm 86.4$ & $184.04 \pm 95.2$ & 0.24 \\
\hline$W C(\mathrm{~cm})$ & $95.86 \pm 10.9$ & $95.55 \pm 10.3$ & $95.18 \pm 10.8$ & $99.29 \pm 7.5$ & 0.13 \\
\hline Total & 602 & 806 & 110 & 43 & -- \\
\hline
\end{tabular}

UA, serum uric acid, MetS, metabolic syndrome; SBP, systolic blood pressure; DBP: diastolic blood pressure; FBG, fasting blood glucose; TG, triglycerides.

Table 4. Correlation Between MetS and UA Levels and HUA in Logistic Models

\begin{tabular}{lccc}
\hline UA Levels $(\mathbf{m g} / \mathbf{d L})$ & Crude OR $(\mathbf{C l ~ 9 5 \% )}$ & $\boldsymbol{P}$ Value & Adjusted OR* $(\mathbf{C l}$ 95\%) \\
\hline$\leq 4.5$ & 1 & - & 1 \\
$4.51-6$ & $0.84(0.66-1.07)$ & 0.159 & $0.91(0.70-1.17)$ \\
$6.01-7$ & $0.93(0.58-1.49)$ & 0.758 & $1.03(0.64-1.67)$ \\
$>7$ & $1.63(0.71-3.75)$ & 0.248 & $1.91(0.82-4.46)$ \\
HUA & $1.33(0.78-2.26)$ & 0.300 & 0.901 \\
\hline
\end{tabular}

UA, serum uric acid; HUA, hyperuricemia; MetS, metabolic syndrome.

*Adjusted by age and gender.

of the variance. They concluded that the contribution of UA as an additional MetS component to explain the total variance seems to be insignificant. They also indicated that the information on UA levels might not be an important factor for exploring the underlying MetS structure. In addition to the MetS main components, there are other factors playing an equally important role in HUA development. Such components include age, BMI, smoking, lifestyle, gender, genetic factors, reduction of endothelial function, increased C-reactive protein activity, which are also associated with HUA pathogenesis. Also, the interrelationship between these factors should not be ignored. Overall, different findings of various studies cannot suggest a consistent role for UA in MetS diagnosis across different study populations.

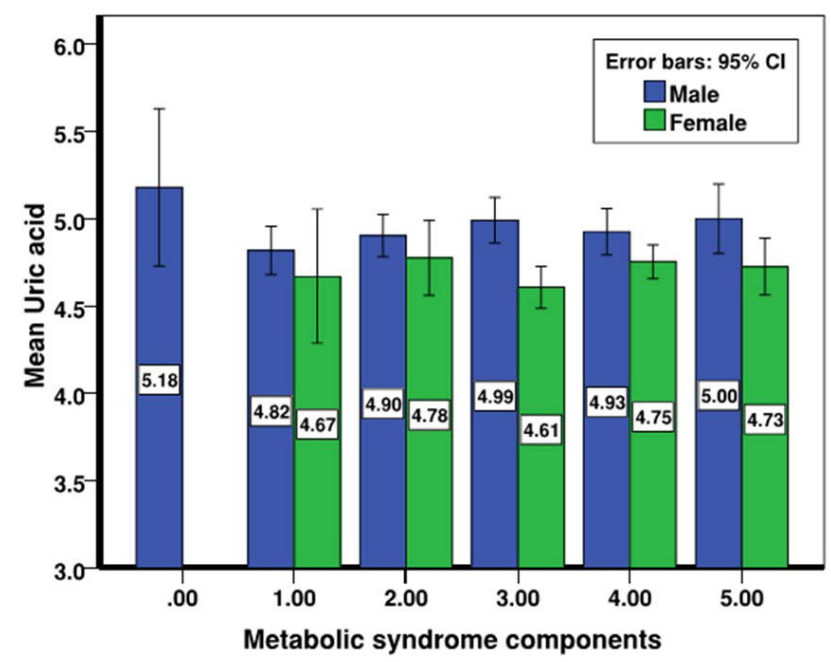

Figure 1. The Association of UA Level With MetS Components by Gender in the Elderly Population.
The role of other important factors in the relationship between UA and MetS should not be ignored. These include the intensity of the disorder among Mets components, the disorder existing simultaneously in the MetS components, whether or not any drugs are taken, and the type of drug if any is being taken. The uricosuric effect of glycosuria in patients with diabetes and fasting hyperglycemia may result in reduced serum UA level in these patients. In addition, the use of antihypertensive agents, especially diuretics, is known to be associated with increased UA levels. ${ }^{41}$

Another concept to explain the difference in results is to use different criteria to define MetS. In Taiwan, the 75 percentile value of WC among middle-aged men is around $88 \mathrm{~cm} .{ }^{43}$ Asian men with $\mathrm{WC} \geq 90 \mathrm{~cm}$ are suggested to have abdominal obesity and to be at risk of comorbidities by the Asian obesity criteria. ${ }^{44}$ By the recently revised ATP III recommendations, WC $\geq 90 \mathrm{~cm}$ was used in Liou et al study for the diagnosis of the MetS. ${ }^{39}$ We used WC $\geq 95 \mathrm{~cm}$ according to the Iranian National Committee of Obesity definition. ${ }^{25}$

The final point is the presence of HUA as symptomatic or asymptomatic. In the studies, it has not been confirmed whether or not the treatment of asymptomatic HUA is effective in improving the treatment of MetS. Some studies do not recommend routine screening for asymptomatic HUA, since treatment does not change the progression of disorders attributed to HUA, such as MetS, diabetes, kidney disease, especially diabetic nephropathy, the most common cause of CKD. ${ }^{45,46}$ There is serious experimental and epidemiological evidence as well as a number of clinical trials to support a relationship of $\mathrm{HUA}$ to $\mathrm{CKD}$, and that $\mathrm{UA}$ lowering treatment might forestall CKD progression. However, these studies were of limited duration and small study population. Therefore, definitive answers about the efficacy and safety of pharmacological treatment for asymptomatic HUA in CKD 
would only be provided using large randomized controlled trials (RCTs). Although there is important evidence that UA lowering drugs highly benefits treating asymptomatic HUA in the setting of CKD, unavailability of these RCTs makes the routine recommendation of these drugs unsubstantiated.

This hypothesis can also be generalized and inclusive for other complications attributed to HUA such as MetS. Although a strong relationship between HUA and MetS is established through animal and epidemiological studies, the potential pathophysiological mechanism through which UA contributes to this disease state is just starting to get clarified. It is apparent that further studies are required to fully understand the role(s) of UA in MetS. ${ }^{46}$ indicates that although HUA is well recognized as a risk factor for atherosclerotic diseases such as myocardial infarction and stroke $\mathrm{e}^{35}$ its independent association with cardiometabolic risk factors remained controversial. ${ }^{11,47}$ Although the reduction in endothelial nitric oxide bioavailability by UA is likely to be involved, the precise biological mechanisms underlying the association between serum UA and the development of MetS remains unclear. Nitric oxide seems to play an important role in the development of insulin resistance. Its deficiency is believed to reduce blood flow to insulin-sensitive tissues i.e. skeletal muscle, liver, adipose tissue, leading to block the insulin action..$^{21,48,49}$ Furthermore, HUA reduces urinary UA excretion through the effect of insulin on urinary tubules leading to HUA. ${ }^{50}$

Most of the recent studies conducted on the relationship between UA levels with MetS concluded that no causal relationship actually exists. These results may be explained by some limitations involved including the studies being observational with small sample size. Moreover, the role of insulin resistance, renin-angiotensin system, endothelial dysfunction and oxidative stress were not assessed in these studies.

Although, the relationship between UA levels and cardiovascular conditions has been studied by a number of researches since the $1960 \mathrm{~s},{ }^{47}$ the results remain controversial. Also, the association between MetS and UA levels ranging between normal to high has not been fully investigated. In addition, most studies were conducted primarily on adults and the institutionalized population. There are a limited number of studies focusing on the elderly population which has a higher prevalence of HUA and MetS, and are of higher risk of developing CVD events. Kuzuya et al evaluated the effect of aging on UA levels in a longitudinal study among a large Japanese population group. They showed that the UA serum level is affected by aging, genetic, environmental factors as well as aging-related hormonal changes. ${ }^{33}$ Estrogen hormone plays an important role in the regulation of serum UA through the removal of more urate from the kidney, secondary to reabsorption. . $^{27,51,52}$

The strengths and limitations of our study need to be addressed. This is a population-based cross-sectional study with strict training process and quality assurance programs. Amirkola is a small city located in the north of Iran, in the province of Mazandaran with a population of 26232 residents. The significant characteristics of this city include the homogeneity of its residents' economical and residential status, and lifestyle. This conditions resulted in confounding bias reduction and results in reliability improvement. Our study's response rate was high with no statistically significant difference between participants who completed and those who did not complete the data. There are some limitations in this study which are needed to be considered. First, the nature of the cross-sectional study did not allow us to assess the temporal relationship. Also, in order to verify the results, a cohort study with follow-up data is required. Second, this study only included participants aged $\geq 60$ years; therefore, findings may not be generalizable to young people.

\section{Conclusions}

In conclusion, UA levels and HUA were not associated with MetS in elderly people due to a few issues. First of all, UA can be related to the MetS components individually, without being associated with MetS diagnosis. Moreover, a number of MetS components and their disorder severity and whether or not medicine is being consumed for the disrupted component can all affect the end results. MetS can present with varying phenotypes, and this also affects its association with UA. Also, using various definitions for MetS is another reason. In this research, we used the Iranian National Committee of obesity definition for WC. The last point is the level of UA. Different studies used a normal range of UA, its elevated range or both. When utilizing elevated UA range, studies need to indicate if HUA was symptomatic or asymptomatic. This is because the treatment of asymptomatic HUA is not confirmed in MetS.

However, with various confounders included metabolic and nonmetabolic components in the association between MetS and UA, which in turn fits into an intricate web of feedbacks, and the inevitable limitations in our understanding of vascular physiology, the whole relationship remains to be determined. Therefore, well-design studies of cohort and clinical trials are needed to assess the association in the community elderly population.

\section{Authors' Contributions}

$A f B$ and $M B$ have made contributions towards the design and execution. SRH and ED have participated in laboratory data collection. AIB has helped to data analysis.

\section{Conflict of Interest Disclosures}

The authors declare they have no conflicts of interest.

\section{Acknowledgments}

Authors thank all the participants for their cooperation and Babol University of Medical Sciences, which provided the financial support for this project.

\section{References}

1. Guarner-LansV, Rubio-Ruiz ME, Pérez-Torres I, Baños de MacCarthy G. Relation of aging and sex hormones to metabolic syndrome and cardiovascular disease. Exp Gerontol. 2011;46(7):517-523. doi:10.1016/j.exger.2011.02.007.

2. Fajemiroye JO, da Cunha LC, Saavedra-Rodríguez R, et al. AgingInduced Biological Changes and Cardiovascular Diseases. Biomed Res Int. 2018;2018:7156435. doi:10.1155/2018/7156435.

3. Veronica G, Esther RR. Aging, metabolic syndrome and the heart. Aging Dis. 2012;3(3):269-279. 
4. Hoffman EL, VonWald T, Hansen K. The metabolic syndrome. S D Med. 2015;Spec No:24-28.

5. Mokhayeri $Y$, Riahi SM, Rahimzadeh S, Pourhoseingholi MA, Hashemi-Nazari SS. Metabolic syndrome prevalence in the Iranian adult's general population and its trend: A systematic review and meta-analysis of observational studies. Diabetes Metab Syndr. 2018;12(3):441-453. doi:10.1016/j.dsx.2017.12.023.

6. Pasalic D, Marinkovic N, Feher-Turkovic L. Uric acid as one of the important factors in multifactorial disorders--facts and controversies. Biochem Med (Zagreb). 2012;22(1):63-75. doi:10.11613/BM.2012.007.

7. Souza-Junior T, Lorenco-Lima L, Ganini D, Vardaris C, Polotow T, Barros M. Delayed uric Acid accumulation in plasma provides additional anti-oxidant protection against iron-triggered oxidative stress after a wingate test. Biol Sport. 2014;31(4):271-276. doi:10.5604/20831862.1120934.

8. Corry DB, Eslami P, Yamamoto K, Nyby MD, Makino H, Tuck ML. Uric acid stimulates vascular smooth muscle cell proliferation and oxidative stress via the vascular renin-angiotensin system. J Hypertens. 2008;26(2):269-275. doi:10.1097/ HJH.0b013e3282f240bf.

9. Kanellis J, Kang DH. Uric acid as a mediator of endothelial dysfunction, inflammation, and vascular disease. Semin Nephrol. 2005;25(1):39-42. doi:10.1016/j.semnephrol.2004.09.007.

10. Khichar S, Choudhary S, Singh VB, Tater P, Arvinda RV, Ujjawal V. Serum uric acid level as a determinant of the metabolic syndrome: A case control study. Diabetes Metab Syndr. 2017;11(1):19-23. doi:10.1016/j.dsx.2016.06.021.

11. Acevedo A, Benavides J, Chowdhury M, et al. Hyperuricemia and cardiovascular disease in patients with hypertension. Conn Med. 2016; 80:85-90.

12. Khan AA, Quinn TJ, Hewitt J, Fan Y, Dawson J. Serum uric acid level and association with cognitive impairment and dementia: systematic review and meta-analysis. Age (Dordr). 2016;38(1):16. doi:10.1007/s11357-016-9871-8.

13. Chou YC, Kuan JC, Yang T, et al. Elevated uric acid level as a significant predictor of chronic kidney disease: a cohort study with repeated measurements. J Nephrol. 2015;28(4):457-462. doi:10.1007/s40620-014-0158-9.

14. Nasri K, Razavi M, Rezvanfar MR, Mashhadi E, Chehrei A, Mohammadbeigi A. Mid-gestational serum uric acid concentration effect on neonate birth weight and insulin resistance in pregnant women. Int J Crit Illn Inj Sci. 2015;5(1):17-20. doi:10.4103/22295151.152309

15. Lee JM, Kim HC, Cho HM, Oh SM, Choi DP, Suh I. Association between serum uric acid level and metabolic syndrome. J Prev Med Public Health. 2012;45(3):181-187. doi:10.3961/ jpmph.2012.45.3.181.

16. Nejatinamini S, Ataie-Jafari A, Qorbani $M$, et al. Association between serum uric acid level and metabolic syndrome components. J Diabetes Metab Disord. 2015;14:70. doi:10.1186/ s40200-015-0200-z.

17. Mukhopadhyay P, Ghosh S, Pandit K, Chatterjee P, Majhi B, Chowdhury S. Uric acid and its correlation with various metabolic parameters: A population-based study. Indian J Endocrinol Metab. 2019;23(1):134-139. doi:10.4103/ijem.IJEM_18_19.

18. Ahmadnezhad M, Arefhosseini SR, Parizadeh MR, et al. Association between serum uric acid, high sensitive C-reactive protein and prooxidant-antioxidant balance in patients with metabolic syndrome. Biofactors. 2018;44(3):263-271.doi:10.1002/biof.1424.

19. Safiri S, Qorbani M, Heshmat R, et al. Association of serum uric acid with cardiometabolic risk factors and metabolic syndrome in Iranian Adolescents: the CASPIAN-III Study. Iran J Kidney Dis. 2016;10(3):126-134

20. Bagheri B, Zargari M, Meshkini F, et al. Uric acid and coronary artery disease, two sides of a single coin: a determinant of antioxidant system or a factor in metabolic syndrome. J Clin Diagn Res. 2016;10(2):Oc27-31. doi:10.7860/jcdr/2016/16335.7281.
21. Iraj B, Feizi A, Abdar-Esfahani $M$, et al. Serum uric acid level and its association with cardiometabolic risk factors in prediabetic subjects. J Res Med Sci. 2014;19(3):262-267.

22. Ebrahimpour P, Fakhrzadeh H, Heshmat R, Bandarian F, Larijani B. Serum uric acid levels and risk of metabolic syndrome in healthy adults. Endocr Pract. 2008;14(3):298-304. doi:10.4158/ ep.14.3.298.

23. Chiou WK, Wang MH, Huang DH, Chiu HT, Lee YJ, Lin JD, The relationship between serum uric acid level and metabolic syndrome: differences by sex and age in Taiwanese. J Epidemiol. 2010;20(3):219-224. doi:10.2188/jea.je20090078.

24. Bijani A, Ghadimi R, Mikaniki E, et al. Cohort Profile Update: The Amirkola Health and Ageing Project (AHAP). Caspian J Intern Med. 2017;8(3):205-212. doi:10.22088/cjim.8.3.205.

25. Azizi F, Hadaegh F, Khalili D, et al. Appropriate definition of metabolic syndrome among Iranian adults: report of the Iranian National Committee of Obesity. Arch Iran Med. 2010;13(5):426428.

26. Grassi D, Ferri L, Desideri G, et al. Chronic hyperuricemia, uric acid deposit and cardiovascular risk. Curr Pharm Des. 2013;19(13):2432-2438. doi:10.2174/1381612811319130011.

27. Abbasian M, Ebrahimi H, Delvarianzadeh M, Norouzi P, Fazli M. Association between serum uric acid (SUA) levels and metabolic syndrome (MetS) components in personnel of Shahroud University of Medical Sciences. Diabetes Metab Syndr. 2016;10(3):132-136. doi:10.1016/j.dsx.2016.01.003.

28. Yuan H, Yu C, Li X, et al. Serum uric acid levels and risk of metabolic syndrome: a dose-response meta-analysis of prospective studies. J Clin Endocrinol Metab. 2015;100(11):4198-4207. doi:10.1210/ jc.2015-2527.

29. Stelmach MJ, Wasilewska N, Wicklund-Liland LI, Wasilewska A. Blood lipid profile and BMI-Z-score in adolescents with hyperuricemia. Ir J Med Sci. 2015;184(2):463-468. doi:10.1007/ s11845-014-1146-8.

30. Krzystek-Korpacka M, Patryn E, Kustrzeba-Wojcickal, Chrzanowska J, Gamian A, Noczynska A. Gender-specific association of serum uric acid with metabolic syndrome and its components in juvenile obesity. Clin Chem Lab Med. 2011;49(1):129-136. doi:10.1515/ cclm.2011.011.

31. Kong AP, Choi KC, Ho CS, et al. Associations of uric acid and gamma-glutamyltransferase (GGT) with obesity and components of metabolic syndrome in children and adolescents. Pediatr Obes. 2013;8(5):351-357. doi:10.1111/j.2047-6310.2012.00115.x.

32. Cardoso AS, Gonzaga NC, Medeiros CC, Carvalho DF. Association of uric acid levels with components of metabolic syndrome and non-alcoholic fatty liver disease in overweight or obese children and adolescents. J Pediatr (Rio J). 2013;89(4):412-418. doi:10.1016/j.jped.2012.12.008.

33. Kuzuya M, Ando F, Iguchi A, Shimokata H. Effect of aging on serum uric acid levels: longitudinal changes in a large Japanese population group. J Gerontol A Biol Sci Med Sci. 2002;57(10):M660-664. doi:10.1093/gerona/57.10.m660.

34. Culleton BF, Larson MG, Kannel WB, Levy D. Serum uric acid and risk for cardiovascular disease and death: the Framingham Heart Study. Ann Intern Med. 1999;131(1):7-13. doi:10.7326/00034819-131-1-199907060-00003.

35. Moriarity JT, Folsom AR, Iribarren C, Nieto FJ, Rosamond WD. Serum uric acid and risk of coronary heart disease: Atherosclerosis Risk in Communities (ARIC) Study. Ann Epidemiol. 2000;10(3):136143. doi:10.1016/S1047-2797(99)00037-X.

36. Leyva F, Godsland IF, Worthington M, Walton C, Stevenson JC. Factors of the metabolic syndrome: baseline interrelationships in the first follow-up cohort of the HDDRISC Study (HDDRISC-1). Heart Disease and Diabetes Risk Indicators in a Screened Cohort. Arterioscler Thromb Vasc Biol. 1998;18(2):208-214. doi:10.1161/01.atv.18.2.208.

37. Donahue RP, Bean JA, Donahue RD, Goldberg RB, Prineas RJ. Does insulin resistance unite the separate components of the 
insulin resistance syndrome? Evidence from the Miami Community Health Study. Arterioscler Thromb Vasc Biol. 1997;17(11):24132417. doi:10.1161/01.atv.17.11.2413.

38. Hodge AM, Boyko EJ, de Courten M, et al. Leptin and other components of the metabolic syndrome in mauritius--a factor analysis. Int J Obes Relat Metab Disord. 2001;25(1):126-131. doi:10.1038/sj.ijo.0801522.

39. Liou TL, Lin MW, Hsiao LC, et al. Is hyperuricemia another facet of the metabolic syndrome? J Chin Med Assoc. 2006;69(3):104-109. doi:10.1016/s1726-4901(09)70186-8.

40. Viazzi F, Leoncini G, Vercelli M, Deferrari G, Pontremoli R. Serum uric acid levels predict new-onset type 2 diabetes in hospitalized patients with primary hypertension: the MAGIC study. Diabetes Care. 2011;34(1):126-128. doi:10.2337/dc10-0918.

41. Aguilar-Andrade G, Álvarez-Bañuelos TM, Morales-Romero J, Fonseca-Reyes S, Margozzini-Maira P, Carvajal-Moreno M. Relationship between uric acid and metabolic syndrome among users of a primary care clinic in Mexico. Metabolomics (Los Angel). 2018;8(1):197. doi:10.4172/2153-0769.1000197.

42. Meigs JB. Invited commentary: insulin resistance syndrome? Syndrome $X$ ? Multiple metabolic syndrome? A syndrome at all? Factor analysis reveals patterns in the fabric of correlated metabolic risk factors. Am J Epidemiol. 2000;152(10):908-911; discussion 912. doi:10.1093/aje/152.10.908.

43. Teh $\mathrm{BH}, \mathrm{Pan} \mathrm{WH}$, Chen $\mathrm{CJ}$. The reallocation of body fat toward the abdomen persists to very old age, while body mass index declines after middle age in Chinese. Int J Obes Relat Metab Disord. 1996;20(7):683-687.

44. Hong AR, Lim S. Clinical characteristics of metabolic syndrome in Korea, and its comparison with other Asian countries. J Diabetes Investig. 2015;6(5):508-515. doi:10.1111/jdi.12313.
45. Chen HS. Clinical implications of the metabolic syndrome and hyperuricemia. J Chin Med Assoc. 2011;74(12):527-528. doi:10.1016/j.jcma.2011.10.005.

46. Soltani Z, Rasheed K, Kapusta DR, Reisin E. Potential role of uric acid in metabolic syndrome, hypertension, kidney injury, and cardiovascular diseases: is it time for reappraisal? Curr Hypertens Rep. 2013;15(3):175-181. doi:10.1007/s11906-013-0344-5.

47. Liu M, He $Y$, Jiang $B$, et al. Association between serum uric acid level and metabolic syndrome and its sex difference in a Chinese community elderly population. Int J Endocrinol. 2014;2014:754678. doi:10.1155/2014/754678.

48. Khomarlou N, Aberoomand-Azar P, Lashgari AP, et al. Essential oil composition and in vitro antibacterial activity of Chenopodium album subsp. striatum. Acta Biol Hung. 2018;69(2):144-155. doi:10.1556/018.69.2018.2.4.

49. Sansbury BE, Hill BG. Regulation of obesity and insulin resistance by nitric oxide. Free Radic Biol Med. 2014;73:383-99. doi:10.1016/j.freeradbiomed.2014.05.016.

50. Gersch C, Palii SP, Kim KM, Angerhofer A, Johnson RJ, Henderson GN. Inactivation of nitric oxide by uric acid. Nucleosides Nucleotides Nucleic Acids. 2008;27(8):967-978. doi:10.1080/15257770802257952.

51. Basaran N, Evliyaoğlu O, Sucu V, et al. Changing of uric acid levels by age and sex in patients with diabetes mellitus. Journal of Clinical and Experimental Investigations. 2016;7(1):1-6. doi:10.5799/ahinjs.01.2016.01.0561.

52. Zhang X, Zhu C, Gao J, et al. Gender difference in the relationship between serum uric acid reduction and improvement in body fat distribution after laparoscopic sleeve gastrectomy in Chinese obese patients: a 6-month follow-up. Lipids Health Dis. 2018;17(1):288. doi:10.1186/s12944-018-0934-y. 